\title{
Avaliação da capacidade dessecante de duas zeolitas obtidas da modificação hidrotérmica de um caulim vermelho do Ceará, Brasil
}

\section{(Evaluation of desiccant capacity of two zeolites obtained from hydrothermal modification of a red kaolin from Ceará, Brazil)}

\author{
J. H. S. Girão, L. G. C. Maia, L. R. D. da Silva \\ Laboratório de Catálise e Materiais \\ Departamento de Química Orgânica e Inorgânica \\ Universidade Federal do Ceará \\ C.P. 6002, Campus do Pici, Fortaleza, Ceará, Brasil, 60451-970
}

\begin{abstract}
Resumo
As zeolitas $\mathrm{P}$ e phillipsita obtidas previamente, a partir de reações hidrotérmicas em meio alcalino de um caulim vermelho do Ceará, foram submetidas a avaliações quanto à capacidade dessecante aplicadas ao solvente acetato de etila contendo quantidades de água que variam de 0,1 a $1 \% \mathrm{v} / \mathrm{v}$. Os resultados mostram que ambas zeolitas apresentam boa capacidade dessecante, tendo a phillipsita alcançado um desempenho notadamente superior ao da zeolita $\mathrm{P}$.

Palavras-chave: dessecante, acetato de etila, zeolita P, phillipsita.
\end{abstract}

\section{Abstract}

The zeolites $P$ e phillipsite previously obtained from hydrothermal reactions in alkaline media of a red kaolin from Ceará, Brazil, were evaluated taking into account the desiccant capacity in ethyl acetate solvents with water in the $0.1-1 \%$ vol. range The results show that both zeolites presented good desiccant capacity, with phillipsite having superior performance.

Keywords: drying agent, ethyl acetate, zeolite P, phillipsite.

\section{INTRODUÇÃO}

Em virtude das impurezas normalmente encontradas nos solventes comerciais, tais como ácidos, bases, peróxidos, água, graxas, entre outras, torna-se necessário o uso de métodos que possam removê-los. Em alguns casos, procedimentos físicos simples como a destilação, são suficientes [1].

Um método muito difundido e de comprovada eficácia é o que emprega dessecantes, para auxiliar na remoção de água de solventes nos quais ela é solúvel [2].

A eficiência desses dessecantes está intimamente relacionada com as suas estruturas, e com a afinidade química em relação à água. Por essa razão, a escolha do dessecante apropriado é um passo importante para o êxito da secagem, pois existem aqueles que agem por meio de adsorção, como a sílica gel; outros atuam através de absorção, como as zeolitas (peneiras moleculares), ou ainda como o sódio metálico que age através de reações químicas $[2,3]$.

Dentre os vários dessecantes encontrados no mercado, as zeolitas ocupam lugar de destaque na secagem de diversos solventes, dentre eles os mais utilizados sendo acetato de etila, benzeno, dioxana, acetonitrila, acetona, piridinas, HMPT, DMSO, DMF e alguns álcoois [4].

Os tipos de zeolitas sintéticas mais adequados para o uso como dessecantes, ou na purificação de gases, são as zeolitas $3 \mathrm{~A}, 4 \mathrm{~A}, 5 \mathrm{~A}$ e $13 \mathrm{X}$ [3]. Estima-se que cerca de $17 \%$ de todas as aplicações para as quais as zeolitas estão voltadas, é como dessecantes. Certamente uma das grandes vantagens de sua utilização está na possibilidade de recuperação, a partir da qual se pode utilizar para um mesmo solvente, cerca de 5000 vezes, que leva a uma perda de eficiência da ordem de apenas $35 \%$, representando uma grande economia de material.

Uma discussão sobre a secagem de solventes empregando várias substâncias inorgânicas, bem como a zeolita A, com capacidade superior em relação aos demais dessecantes foi apresentada [3].

Estudos tem mostrado a eficiência desses materiais para diversos solventes, que tem revelado particularidades no comportamento dos vários secantes encontrados no mercado [2-4].

Este trabalho visa avaliar a eficiência de dois tipos zeolíticos sintetizados a partir de um caulim vermelho do Ceará $[5,6]$. A escolha do solvente acetato de etila, se deve ao grande volume deste que tem sido utilizado no laboratório de catálise e materiais da Universidade Federal do Ceará. As zeolitas utilizadas foram as formas sintéticas zeolita $\mathrm{P}$ e phillipsita.

\section{MATERIAISE MÉTODOS}

Inicialmente foram preparadas três amostras de acetato de etila (Reagen), variando-se a quantidade de água no mesmo, desde $0,1 \% \mathrm{v} / \mathrm{v}$ a $1 \% \mathrm{v} / \mathrm{v}$. Foi acrescentado alaranjado de acridina (3,6-bisdimetilaminoacridina) em quantidades suficientes para 
saturar as soluções, seguido de medidas a $490 \mathrm{~nm}$ em um espectrofotômetro Hitashi.

Amostras das zeolitas $\mathrm{P}$ e phillipsita foram calcinadas em mufla a $250^{\circ} \mathrm{C}$, durante 11 horas, e resfriadas em dessecador sob vácuo. Foram colocados $100 \mathrm{mg}$ de zeolita em erlenmeyer, em seguida foram acrescentados $10 \mathrm{~mL}$ de acetato de etila a 1 $\% \mathrm{v} / \mathrm{v}$, e deixados sob agitação durante 24 horas. Ao final desse período o solvente foi separado da zeolita por centrifugação, saturado com o alaranjado de acridina e analizado a $490 \mathrm{~nm}$.

\section{RESULTADOS E DISCUSSÃO}

Os resultados das análises baseiam-se na reação entre a água e o alaranjado de acridina [7]. Este na sua forma neutra, ao interagir com a água resulta num deslocamento do equilíbrio ácido-base, levando à forma monoprotônica, como mostra a equação química a seguir.

Além da autoionização da água é também possível a

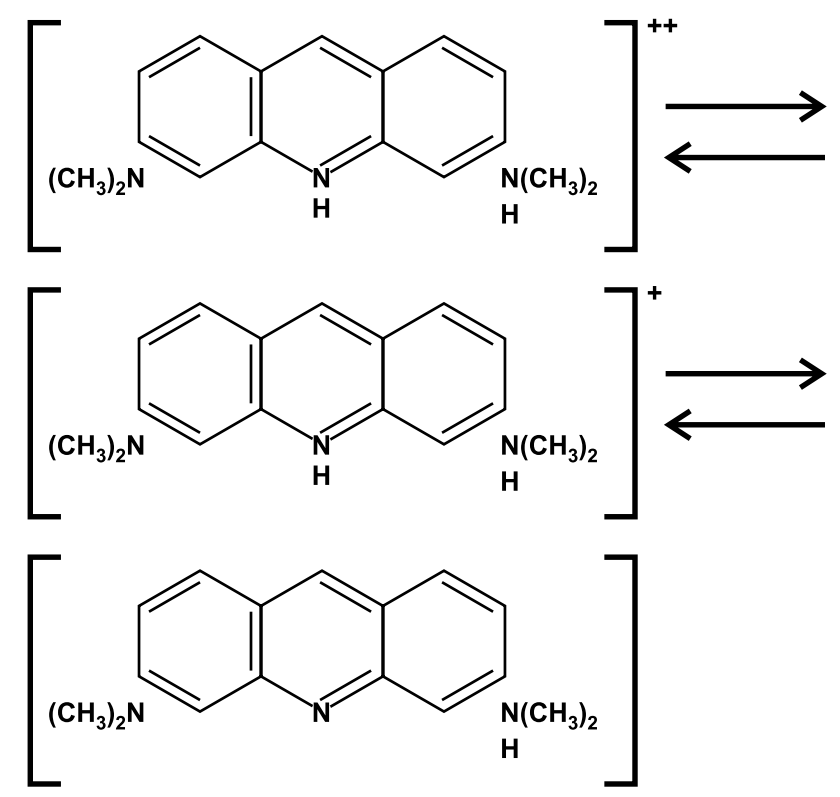

autoionização do solvente, e com isso, a quantidade da espécie $\mathrm{H}^{+}$torna-se muito pequena, permitindo com isso, isolar a espécie neutra (III) do corante em solventes orgânicos, determinandose a quantidade de água presente no meio orgânico, através da banda em $490 \mathrm{~nm}$ relativa à espécie monoprotonada (II) [8].

As medidas espectrofotométricas resultaram nos espectros da Fig. 1, que confirmam a formação da espécie II. Observa-se também, que para o solvente contendo $1 \% \mathrm{v} / \mathrm{v}$ de água surge uma banda atribuída à espécie III. Existe um ponto isosbéstico em $390 \mathrm{~nm}$ que indica a presença de um único equilíbrio entre as espécies II e III. O aparecimento desse ponto indica também, que não existem dímeros do alaranjado de acridina na solução, nessa concentração.

O gráfico da Fig. 2 mostra uma correlação linear de 0,99 entre as medidas feitas para as soluções de $0,1,0,5$ e 1,0\%v/v em água. A partir desse resultado foi selecionada a solução a $1 \% \mathrm{v} / \mathrm{v}$ em água para as avaliações das zeolitas como

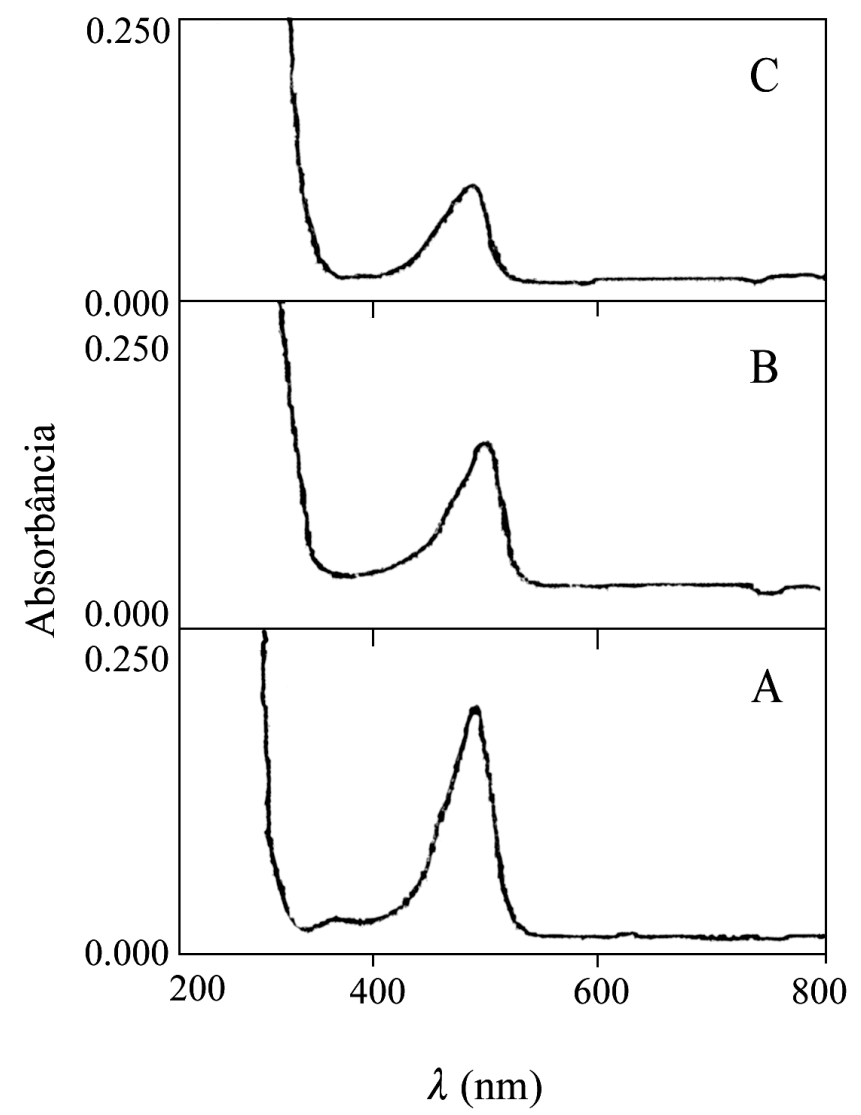

Figura 1: Espectros de absorção UV-Vis do complexo alaranjado de acridinaágua em acetato de etila. (A) $1 \% \mathrm{v} / \mathrm{v}$; (B) $0,5 \% \mathrm{v} / \mathrm{v}$; (C) $0,1 \% \mathrm{v} / \mathrm{v}$. [Figure 1: Absorption spectras UV-Vis by acridine orange-water in ethyl acetate. (A) $1 \% \mathrm{v} / \mathrm{v}$; (B) $0.5 \% \mathrm{v} / \mathrm{v}$; (C) $0.1 \% \mathrm{v} / \mathrm{v}$.]

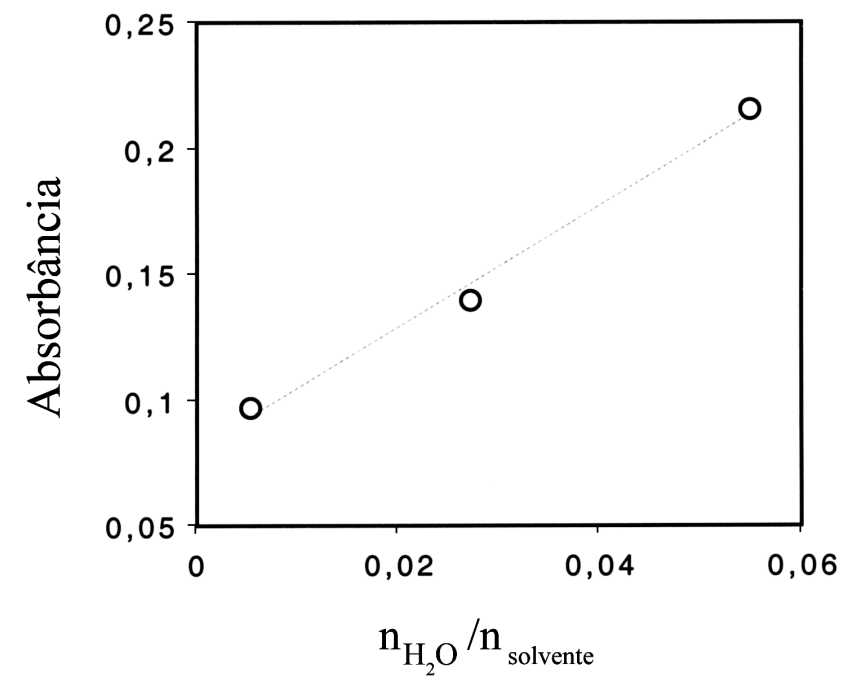

Figura 2: Correlação linear para a concentração molar de água presente no solvente acetato de etila.

[Figure 2: Linear correlation for the molar concentration of water in ethyl acetate solvent.]

dessecantes, tomando-se o cuidado de empregar uma relação $\mathrm{m}_{\mathrm{H} 2 \mathrm{O}} / \mathrm{m}_{\mathrm{ZEO}}$, suficientemente elevada, para permitir que ao final se tivesse água residual no solvente, para produzir a complexação com o alaranjado de acridina. 


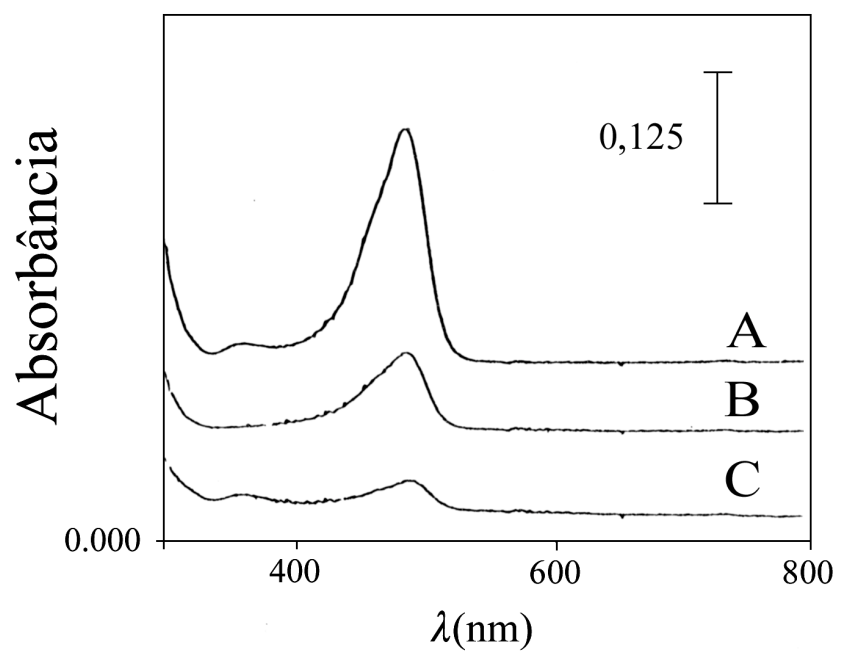

Figura 3: Espectros de absorção UV-Vis para o solvente tratado com os dessecantes. (A) Antes da secagem; (B) Após secagem com a Zeolita P; (C) Após secagem com a Phillipsita.

[Figure 3: UV-Vis absorption spectra for the solvent drying desiccants. (A) Before drying; (B) After drying zeolite P; (C) After drying Phillipsite.]

Após a utilização das zeolitas na secagem do solvente, foi determinada a quantidade de água a partir dos espectros de absorção, como mostra a Fig. 3 .

Tais resultados indicam que a zeolita $\mathrm{P}$, espectro $\mathrm{B}$, foi capaz de reter cerca de $0,68 \mathrm{~g}$ de água para cada grama de zeolita. Enquanto que a phillipsita, espectro $\mathrm{C}$, reteve cerca de $0,87 \mathrm{~g}$ de água por grama de zeolita. Comparando esses resultados com os de outros tipos de dessecantes, observa-se que mesmo para a zeolita $\mathrm{P}$, a capacidade dessecante pode ser considerada elevada [3].

\section{CONCLUSÕES}

A eficiência demonstrada pelas zeolitas P e phillipsita confirma sua maior capacidade como dessecantes, quando comparada aos resultados encontrados na literatura para diversos secantes de uso freqüente nos laboratórios de síntese orgânica. Este trabalho mostrou também que o método espectrofotométrico do alaranjado de acridina pode ser empregado de maneira bastante simples, como já sugerido [8].

\section{AGRADECIMENTOS}

Os autores agradecem a Funcap e Universidade Federal do Ceará pelo financiamento desta pesquisa.

\section{REFERÊNCIAS}

[1] D. D. Perrin, W. L. P. Armarego, "Purification of laboratory chemicals", $3^{\text {rd }}$ Ed., Pergamon Press, New York (1988) 4.

[2] L. F. Fieser, "Reagents for organic synthesis", John Wiley \& Sons, New York (1967) 703.

[3] V. F. Ferreira, Química Nova 15 (1992) 348.

[4] V. Sedivee, J. Flek, "Handbook of analysis of organic solvents", John Wiley \& Sons, New York (1976) 16.

[5] J. H. S. Girão, L. R. D. da Silva, Mater. Lett. (2001), no prelo.

[6] J. H. S. Girão, M. A. B. de Araújo, L. R. D. da Silva, Mater. Chem. Phys. (2001), no prelo.

[7] M. Guzman, J. L. P. Pavon, E. R. Gonzalo, C. Hatfield, J. Ruzicha, G. D. Christian, Analyst, 116 (1991) 1043.

[8] E. Sabadini, R. A. Bariccatti, F. B. T. Pessine, T. D. Z. Atvars, Química Nova 17 (1994) 27.

(Rec. 26/12/01, Ac. 01/11/02) 